\title{
Desigualdades na tendência da sífilis congênita no município de Niterói, Brasil, 2007 a 2016
}

\author{
Andressa Lohan dos Santos Heringer, ${ }^{1}$ Helia Kawa, ${ }^{1}$ Sandra Costa Fonseca, ${ }^{1}$ Sandra Mara Silva \\ Brignol, ${ }^{1}$ Loren Angelica Zarpellon ${ }^{1}$ e Ana Cristina Reis ${ }^{2}$
}

Como citar Heringer ALS, Kawa H, Fonseca SC, Brignol SMS, Zarpellon LA, Reis AC. Desigualdade na tendência da sífilis congênita no município de Niterói, Brasil 2007 a 2016. Rev Panam Salud Publica. 2020;44:e8. https://doi.org/10.26633/RPSP.2020.8

RESUMO Objetivo. Descrever a distribuição temporal e as características epidemiológicas da sífilis congênita (SC) em
Niterói, Sudeste do Brasil, de 2007 a 2016 .
Métodos. Este estudo descritivo de série temporal da incidência de SC utilizou os dados do Sistema de Infor-
mação de Agravos de Notificação (SINAN) e do Sistema de Informações sobre Nascidos Vivos (SINASC). A
amostra incluiu todos os casos notificados. Além disso, foi realizado um relacionamento probabilístico entre
SINAN e SINASC para recuperar informações ignoradas. A série temporal foi estimada por regressão logarít-
mica, de acordo com variáveis sociodemográficas e de pré-natal.
Resultados. Identificaram-se 754 casos de SC no período estudado (incidência média de 11,9 casos/1 000
nascidos vivos). A incidência foi mais elevada em jovens (10 a 19 anos; 20 a 24 anos), participantes de cor
preta e naquelas com baixa escolaridade e sem pré-natal. Do total de mulheres, apenas $57,6 \%$ obtiveram o
diagnóstico de sífilis durante o pré-natal. O tratamento foi inadequado em $87,7 \%$ das mulheres. Apenas $12,2 \%$
dos parceiros foram tratados. Houve tendência crescente do agravo (16\%/ano), que atingiu 23,2 casos/1 000
nascidos vivos em 2016 . O crescimento foi mais acentuado em adolescentes do sexo feminino (25,2\%/ano),
raça/cor parda (16,8\%/ano), indivíduos com baixa escolaridade ( $57,1 \% / a n o)$ e mulheres que realizaram
pré-natal (17,3\%/ano); e, no período de 2012 a 2016 , em mulheres com informação ignorada para a cor da pele.
Conclusões. As iniquidades sociais se destacaram na ocorrência de SC, com incidência crescente em
jovens. É necessária a capacitação dos profissionais de saúde para o manejo da sífilis gestacional e uma
atuação efetiva das políticas públicas sobre os determinantes sociais da sífilis.

A sífilis congênita (SC) é uma doença que pode ser evitada pelo diagnóstico correto e pelo subsequente tratamento da gestante infectada (1). No entanto, dificuldades no diagnóstico oportuno e no tratamento adequado de gestantes e de seus parceiros $(2,3)$ têm levado a um aumento da sífilis em gestantes, com consequente aumento da transmissão vertical (4).

Nesse contexto, a Organização Pan-Americana da Saúde (OPAS) propôs renovar e articular as estratégias de enfrentamento da SC, definindo como meta para o ano de 2020 uma incidência de 0,5 casos / 1000 nascidos vivos (5). Para alcançar essa meta, é preciso implementar ações interprogramáticas que aumentem a cobertura da testagem de gestantes e mulheres em idade fértil e o tratamento imediato dos casos positivos $(5,6)$.

Mundialmente, a incidência de SC diminuiu de 5,4 casos por 1000 nascidos vivos, em 2012, para 4,7 em 2016, exceto na Região das Américas e no Leste do Mediterrâneo (7). Em 2016, as maiores taxas se encontravam no continente africano $(12 / 1000$ nascidos vivos) e as menores, na Europa (0,2/1 000 nascidos vivos) (7). Nas Américas, houve aumento de 3,07 para 3,19/1 000 nascidos vivos de 2012 a 2016 (7), embora alguns países tenham

\footnotetext{
1 Universidade Federal Fluminense (UFF), Instituto de Saúde Coletiva, Departamento de Epidemiologia e Bioestatística, Niterói (RJ), Brasil. $\square$ Andressa Lohan dos Santos Heringer, andressa_lohan@hotmail.com
} 
conseguido o certificado de eliminação da transmissão vertical de SC (5).

Na América Latina, o Brasil é responsável por 85\% dos casos de SC (5), com aumento consistente ao longo dos anos (8): em 2007, a taxa era de 1,9 caso/1 000 nascidos vivos; em 2017, chegou a 8,6 casos / 1000 nascidos vivos, com 24666 casos notificados (8). Naquele ano, a região Sudeste do país apresentou a maior taxa de incidência. Foi destaque o estado do Rio de Janeiro, com 18,8 casos/1 000 nascidos vivos (8) e taxas ainda mais altas em suas duas regiões metropolitanas: 22,1/1 000 nascidos vivos na Metropolitana I, que engloba a capital do estado e os municípios próximos, situados na baixada fluminense; e 26,9/1 000 nascidos vivos na Metropolitana II, também formada por municípios próximos da capital, porém do outro lado da Baía de Guanabara. Juntas, as regiões Metropolitanas I e II comportam três quartos da população do estado (9).

Os dados atuais sobre a SC no Brasil são provenientes do Sistema de Informação de Agravos de Notificação (SINAN), que apresenta subnotificação e elevado percentual de informações ignoradas ou em branco (4, 10-12). Isso limita o uso desse sistema em estudos sobre incidência e fatores relacionados ao aumento da SC. Entretanto, esse déficit pode ser compensado pelo relacionamento com outros sistemas de informação em saúde, como o Sistema de Informações sobre Nascidos Vivos (SINASC), o Sistema de Informações sobre Mortalidade (SIM) e o Sistema de Informações Hospitalares (SIH), aumentando a completude e a confiabilidade dos dados $(10,13)$. Por sua vez, a análise de séries temporais $(12,13,14)$ pode revelar não só a evolução da incidência, mas também fatores associados às mudanças nos padrões de distribuição do agravo (15), sendo de grande utilidade para agravos de difícil controle, como a SC.

Diante disso, o objetivo deste estudo foi descrever a distribuição temporal e as características epidemiológicas da SC em residentes do município de Niterói, no Sudeste do Brasil, de 2007 a 2016.

\section{MATERIAIS E MÉTODOS}

O presente estudo descritivo, de série temporal, utilizou dados secundários referentes ao município de Niterói, localizado na Região Metropolitana II do estado do Rio de Janeiro, Brasil. O período de análise foi de janeiro de 2007 a dezembro de 2016.

A área territorial do município de Niterói é de 133919 km². A população, estimada em 500000 residentes, é totalmente urbana (16). O município tem índice de desenvolvimento humano (IDH) alto $(0,837)$ e baixa vulnerabilidade social (16). A cobertura populacional da atenção básica era de 67\% em 2007 e de $78 \%$ em 2016 (17).

A população do estudo contemplou todos os casos de SC notificados em residentes no município, incluindo abortos, óbitos fetais e nascidos vivos. Os dados foram obtidos do SINAN, com identificação dos casos de sífilis congênita a partir das fichas de investigação. Essas fichas contêm informações sociodemográficas, epidemiológicas, laboratoriais e clínicas preenchidas por profissionais de saúde. Também foi utilizado o banco de dados do SINASC para complementar as variáveis com respostas ignoradas no SINAN. Quando houve discordâncias entre os sistemas, foi priorizado o SINASC.

Analisaram-se variáveis sociodemográficas maternas faixa etária, raça/cor e escolaridade; e variáveis relativas ao pré-natal/parto - realização do pré-natal (sim/não), momento do diagnóstico de sífilis, esquema de tratamento, testes diagnósticos realizados (teste não treponêmico no parto/curetagem, teste confirmatório treponêmico no parto/curetagem) e tratamento do parceiro. As variáveis idade e escolaridade maternas, raça/cor e número de consultas de pré-natal foram obtidas do SINASC.

O relacionamento entre o SINAN e o SINASC foi realizado pelo programa OpenReclink (18), utilizando chaves de blocagem a partir das variáveis "nome da mãe" e "sexo do bebê" e inserindo, nos campos de comparação, o nome da mãe e a data de nascimento do bebê. Para verificar a veracidade de cada par encontrado, analisaram-se as variáveis bairro de moradia, endereço e idade da mãe, data de nascimento e data da notificação. Foram considerados não pareados os casos em que houve dúvida em pelo menos duas variáveis na identificação da gestante, seja por diferença de nome, idade ou endereço.

A incidência da SC foi calculada pela divisão do número de casos novos por ano pelo número de nascidos vivos no mesmo ano, sendo esse resultado multiplicado por 1 000. O número de nascidos vivos foi obtido do SINASC. A análise estatística descritiva foi realizada para o perfil dos casos incidentes de SC quanto às características sociodemográficas, clínicas e de pré-natal. Estimaram-se medidas de frequência para as variáveis categorizadas e medida de centralidade (mediana) para as variáveis quantitativas utilizando o programa Statistical Package for the Social Sciences (SPSS) versão 20.0.

A análise da tendência temporal foi feita por meio do programa Joinpoint Regression, que permite ajustar uma série de linhas de tendência e seus pontos de junção em uma escala logarítmica, testando as tendências anuais. Foram estimados e testados os percentuais anuais de mudança (annual percent change, APC) e seus respectivos intervalos de confiança de $95 \%$ (IC95\%). Para avaliar a significância estatística, usou-se o teste de permutação de Monte Carlo, que ajusta a melhor linha para cada segmento. Quando não houve mudança, o período foi analisado de forma integral. Para minimizar o efeito de possíveis autocorrelações, utilizou-se a opção disponível no software que permite ajustar um modelo de erros correlacionados com base nos dados (fit an autocorrelated errors model based on the data) (19).

A estimativa da tendência e da variação anual por transformação logarítmica é defendida para reduzir a heterogeneidade da variância na análise de regressão (15). Para essa análise, a categoria "ignorada" também foi usada em algumas variáveis, a fim de avaliar se a sua evolução temporal seria indicadora de algum padrão.

O projeto da pesquisa foi aprovado pelo Comitê de Ética em Pesquisa da Universidade Federal Fluminense (UFF) (parecer 2601802). Durante a realização do estudo, garantiram-se o anonimato e o sigilo dos dados empregados; os princípios de confidencialidade foram respeitados por meio de procedimentos adotados ao longo do processo de relacionamento probabilístico dos dados. As bases identificadas somente incluíam os campos de identificação necessários para o pareamento. As análises foram realizadas nas bases resultantes não identificadas e os resultados foram apresentados de forma agregada. Os bancos de dados do SINAN e do SINASC foram fornecidos, respectivamente, pela Coordenação de Vigilância em Saúde de Niterói (COVIG) e pela Assessoria de Dados Vitais da Secretaria de Estado de Saúde do Rio de Janeiro para utilização no estudo. 


\section{RESULTADOS}

De 2007 a 2016, 754 casos de SC foram notificados e confirmados no município de Niterói, sendo 522 pares identificados nas bases do SINAN e do SINASC. Os 232 casos de SC não pareados por meio do relacionamento foram mantidos na casuística, sendo 51 (21,9\%) classificados como abortamento, 38 (16,4\%) como natimortos, 23 (9,9\%) como óbitos e 120 (51,7\%) como nascidos vivos.

Após o relacionamento das bases de dados, houve diminuição da incompletude das variáveis sociodemográficas, mas algumas se mantiveram com percentual ignorado para mais de $5 \%$ da amostra. A variável escolaridade materna teve a porcentagem de ignorados reduzida de $49,7 \%$ para $18,4 \%$, e a variável cor materna de $7,4 \%$ para $6,3 \%$ após o relacionamento entre os bancos de dados. O não preenchimento também foi elevado para algumas informações exclusivas do SINAN, como esquema de tratamento materno $(10,6 \%)$ e tratamento do parceiro $(28,7 \%)$.

\section{Características das mulheres, do pré-natal e classificação final}

A idade materna variou de 13 a 45 anos (mediana de 22 anos). O maior número de casos de SC ocorreu em filhos de mulheres com menos de 35 anos, com baixa escolaridade $(<8$ anos de estudo), de raça/cor parda e que realizaram pré-natal (tabela 1).

O pré-natal foi realizado por 80,8\% das mulheres; 39,3\% compareceram a sete ou mais consultas. Do total de mulheres, $57,6 \%$ obtiveram o diagnóstico de sífilis durante o pré-natal e as demais souberam da infecção apenas durante a internação para o parto ou curetagem, ou esta informação ficou ignorada (tabela 2).

TABELA 1. Características sociodemográficas de 754 gestantes com desfecho de sífilis congênita, Niterói, Brasil, 2007 a 2016

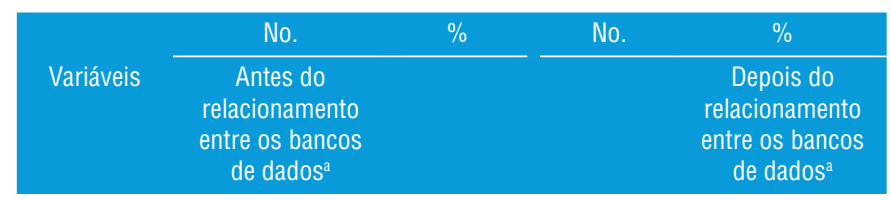

Faixa etária (anos)

\begin{tabular}{lccrr}
10 a 19 & NA & NA & 239 & 31,7 \\
20 a 34 & NA & NA & 461 & 61,1 \\
35 ou mais & NA & NA & 46 & 6,1 \\
$\begin{array}{l}\text { Ignorada } \\
\text { Cor da mãe }\end{array}$ & 8 & 1,1 & 8 & 1 \\
Branca & NA & NA & 138 & 18,3 \\
Preta & NA & NA & 163 & 21,6 \\
Amarela & NA & NA & 1 & 0,1 \\
Parda & NA & NA & 404 & 53,6 \\
Ignorado & 56 & 7,4 & 48 & 6,3 \\
Escolaridade (anos) & & & & \\
$<8$ anos & NA & NA & 473 & 62,7 \\
$>8$ anos & NA & NA & 142 & 18,8 \\
Ignorada & 375 & 49,7 & 139 & 18,4 \\
\hline
\end{tabular}

Fonte: SINAN e SINASC (2007 a 2016).

${ }^{a}$ Relacionamento entre o SINAN e SINASC para recuperar dados ignorados. NA: Não se aplica.
TABELA 2. Características do pré-natal em 754 gestantes com desfecho de sífilis congênita, Niterói, Brasil, 2007 a 2016

\begin{tabular}{|c|c|c|}
\hline Variáveis & No. & $\%$ \\
\hline \multicolumn{3}{|l|}{ Realização de pré-natal } \\
\hline $\operatorname{Sim}$ & 609 & 80,8 \\
\hline Não & 71 & 9,4 \\
\hline Ignorado & 74 & 9,8 \\
\hline \multicolumn{3}{|l|}{ Número de consultas de pré-natal } \\
\hline Nenhuma consulta & 27 & 3,6 \\
\hline 1 a 3 & 42 & 5,6 \\
\hline 4 a 6 & 144 & 19 \\
\hline 7 ou mais & 297 & 39,3 \\
\hline Ignorado & 244 & 32,4 \\
\hline \multicolumn{3}{|l|}{ Diagnóstico de sífilis materna } \\
\hline Durante o pré-natal & 434 & 57,6 \\
\hline No momento do parto/curetagem & 289 & 38,3 \\
\hline Após o parto & 9 & 1,2 \\
\hline Não realizado & 1 & 0,1 \\
\hline Ignorado & 21 & 2,8 \\
\hline \multicolumn{3}{|l|}{ Esquema de tratamento materno ${ }^{\mathrm{a}}$} \\
\hline Adequado & 7 & 1,6 \\
\hline Inadequado & 298 & 68,6 \\
\hline Não realizado & 83 & 19,1 \\
\hline Ignorado & 46 & 10,6 \\
\hline \multicolumn{3}{|l|}{ Parceiro tratado } \\
\hline Não & 445 & 59 \\
\hline $\operatorname{Sim}$ & 92 & 12,2 \\
\hline Ignorado & 217 & 28,7 \\
\hline \multicolumn{3}{|c|}{ Teste não treponêmico no parto/curetagem } \\
\hline Reagente & 705 & 93,5 \\
\hline Não reagente & 29 & 3,8 \\
\hline Não realizado & 7 & 0,9 \\
\hline Ignorado & 13 & 1,7 \\
\hline \multicolumn{3}{|l|}{ Classificação final do caso de SC } \\
\hline Abortamento & 51 & 6,8 \\
\hline Natimorto & 38 & 5 \\
\hline SC recente & 655 & 86,9 \\
\hline SC tardia & 1 & 0,1 \\
\hline Ignorado & 9 & 1,2 \\
\hline
\end{tabular}

Fonte: SINAN e SINASC (2007 a 2016).

a De acordo com as normas vigentes no período estudado (1).

O esquema de tratamento foi inadequado em $68,6 \%$ das mulheres e não realizado em $19,1 \%$, somando $87,7 \%$ das mulheres. Além disso, apenas $12,2 \%$ dos parceiros foram tratados (tabela 2). Quanto à classificação final, predominou SC recente $(86,9 \%)$, seguida de abortos $(6,8 \%)$ e natimortos $(5 \%)$ (tabela 2$)$.

\section{Incidência e série temporal}

A incidência média da sífilis congênita foi de 11,9 casos / 1000 nascidos vivos, aumentando de forma significativa e constante de 2007 (4,3 casos /1 000 nascidos vivos) até 2016 (23,2 casos/1 000 nascidos vivos), com mudança percentual anual de 16\% (IC95\%: 9,9 a 22,4). Em relação à faixa etária, ao final do período, as taxas foram mais elevadas entre as mães adolescentes. Houve intenso e significativo aumento nas mulheres jovens (15 a 19 e 20 a 24 anos), além de aumento moderado naquelas 
TABELA 3. Incidência de sífilis congênita por 1000 nascidos vivos e variação percentual anual conforme variáveis sociodemográficas e de pré-natal em 754 gestantes, Niterói, Brasil

\begin{tabular}{|c|c|c|c|c|c|}
\hline Variáveis & 2007 & 2016 & Período & Mudança percentual anual (IC95\%) & Tendência \\
\hline \multicolumn{6}{|l|}{ Faixa etária (anos) } \\
\hline 10 a 19 & 5,2 & 53,0 & 2007 a 2016 & $25,2^{\mathrm{a}}(12,7$ a 39,1$)$ & Aumento \\
\hline 20 a 24 & 6,3 & 47,9 & 2007 a 2016 & $20,6^{a}(15,0$ a 26,5$)$ & Aumento \\
\hline$\geq 35^{\mathrm{b}}$ & 0,01 & 8,1 & $2008^{\text {b }}$ a 2016 & $-0,0(-11,1$ a 12,4$)$ & Estabilidade \\
\hline \multicolumn{6}{|l|}{ Escolaridade (anos) } \\
\hline$\geq 8$ & 1,2 & 13,7 & 2007 a 2016 & $29,4^{\text {a }}(24,9$ a 34,0$)$ & Aumento \\
\hline \multicolumn{6}{|l|}{ Raça/cor } \\
\hline \multirow[t]{2}{*}{ Ignorada } & 29,4 & 77,4 & 2007 a 2012 & $-20,0(-36.6$ a 1.0) & Estabilidade \\
\hline & & & 2012 a 2016 & $60,7^{\mathrm{a}}(12,9$ a 128,8$)$ & Aumento \\
\hline Branca & 1,7 & 5,4 & 2007 a 2016 & $10,8^{a}(5.3$ a 16,6$)$ & Aumento \\
\hline Ignorada & 70,0 & 101,7 & 2007 a 2016 & $7,8(-0,4$ a 16,6$)$ & Estabilidade \\
\hline $\operatorname{Sim}$ & 3,0 & 20,8 & 2007 a 2016 & $17,3^{\mathrm{a}}(10,0$ a 25,1$)$ & Aumento \\
\hline Não & 14,5 & 126,4 & 2007 a 2016 & $17,6(-5,6$ a 46,4$)$ & Estabilidade \\
\hline
\end{tabular}

Fonte: SINAN e SINASC (2007 a 2016).

a Significância estatística.
${ }^{\mathrm{b}} 0$ ano de 2007 não foi considerado porque ficou como outlier na regressão joinpoint.

com 25 a 34 anos. Apenas a faixa das mais velhas se manteve estável e com a menor taxa de SC (tabela 3). Em relação à escolaridade, todas as faixas indicaram aumento significativo, sendo o maior em mães com baixa escolaridade, embora aquelas para quem essa informação era ignorada tenham atingido a maior taxa em 2016 (tabela 3).

Em relação a raça/cor, as maiores incidências em 2016 foram registradas nas mães com essa informação em branco/ignorada, seguidas das que se autodeclararam pretas (tabela 3). Não houve identificação de tendência significativa de aumento no período de 2007 a 2012 nas categorias de cor preta e ignorada (tabela 3).

Finalmente, em relação à variável da realização de pré-natal, a análise temporal indica que, embora as mães das categorias ignorada e pré-natal não realizado tenham apresentado as incidências mais elevadas, não houve tendência significativa de aumento ( $P=0,1 \mathrm{em}$ ambas as categorias). De forma inversa, observou-se aumento constante e significativo da taxa de SC na categoria de pré-natal realizado (tabela 3).

\section{DISCUSSÃO}

Este estudo identificou que a incidência de SC foi, além de elevada, crescente em Niterói, município que ocupa o sétimo lugar no ranking do IDH no Brasil e que conta com boa cobertura da atenção básica $(16,17)$. A incidência identificada em 2016 (23,2 casos / 1000 nascidos vivos) foi muito distante da meta da Organização Mundial da Saúde (OMS), adotada pela OPAS para a eliminação da SC (0,5 ou menos casos de SC/1 000 nascidos vivos) (5). O aumento observado em Niterói ao longo do tempo acompanhou as características das regiões metropolitanas do estado do Rio de Janeiro e pode ser explicado, em parte, pelo crescimento da sífilis adquirida entre 2013 e 2017, mais acentuado nas faixas etárias de 10 a 19 anos e de 20 a 29 anos (9). O aumento dos casos de sífilis adquirida também tem sido relatado em outras regiões do Brasil (8) e em outros países (5).

A ocorrência de sífilis e SC em gestantes tem sido associada a fatores sociais, econômicos, de infraestrutura e de acesso aos serviços de saúde, acometendo, muitas vezes, populações de maior vulnerabilidade social $(2,11,20)$. Sendo assim, a explicação para os resultados deste estudo pode ser o aumento de iniquidades sociais. As características das mulheres que foram diagnosticadas com SC foram semelhantes às relatadas em outros estudos no território brasileiro, principalmente a baixa escolaridade $(2,13,21-26)$ e a cor da pele parda/preta $(2,11,13$, 21-24). Vale ressaltar que a cidade tem um índice de Gini elevado, inferior apenas ao da capital do Rio de Janeiro (16), e uma cobertura da atenção primária elevada, superior a 70\% na maior parte do período estudado (17). No entanto, iniquidades sociais podem fazer com que mulheres em situação de vulnerabilidade não sejam captadas e acompanhadas de forma adequada pelos serviços de saúde.

O estudo mostrou que a maioria das mulheres $(80,8 \%)$ realizou o pré-natal, como também verificado em outros estados do país e no Distrito Federal (4). Entretanto, apenas 39,3\% compareceram a sete ou mais consultas; ou seja, o acompanhamento recomendado às gestantes com sífilis não foi alcançado (1). A qualidade do pré-natal das mulheres com sífilis gestacional é baixa em todo o país, tanto pelo início tardio $(2,25)$ como pelo número de consultas inferior ao recomendado $(2,11,13,25)$. Um dos reflexos da ausência ou das falhas no pré-natal é o diagnóstico realizado durante a internação para o parto/curetagem ou após o parto em 38,3\% das gestantes residentes em Niterói, corroborando os achados de outras localidades $(4,12,13,26)$. 
Outro estudo realizado em seis estados brasileiros mostrou que no Amazonas, no Ceará e no Rio de Janeiro a maioria das gestantes (59,7\%, 53,3\% e 52,1\%, respectivamente) foi diagnosticada somente durante a internação para o parto (4). No estado do Rio Grande do Sul, de 2001 a 2012, 0,6\% das mulheres não realizou o exame para sífilis no pré-natal, na hora do parto/ curetagem ou após o parto (12).

Além do diagnóstico oportuno da sífilis durante a gestação, o tratamento adequado deve ser instituído imediatamente, a fim de evitar a transmissão vertical (1). No entanto, foi identificada uma porcentagem muito baixa $(1,6 \%)$ de tratamento adequado, resultado ainda inferior ao encontrado em outros estados brasileiros $(4,10,14)$, porém semelhante ao encontrado em Palmas $(1 \%)$, no estado de Tocantins (26). Mundialmente, tanto a incidência da SC como a ocorrência dos desfechos adversos decorrentes da doença estão relacionadas à menor cobertura da atenção pré-natal (7).

Em Niterói, apenas 17\% dos parceiros das gestantes cujo desfecho foi de SC receberam tratamento, falha também identificada em outras localidades do país $(4,11,13,14,24,26)$. Uma pesquisa realizada em Porto Alegre mostrou que, mesmo entre os tratados, apenas $12,4 \%$ receberam o tratamento adequado. Foi identificada, também, relação entre a realização do tratamento do parceiro e a gestante ter mais de 8 anos de estudo, ter realizado o pré-natal adequado e ter recebido o diagnóstico de sífilis durante o pré-natal (3). É importante salientar que, quando o parceiro não é tratado, pode ocorrer a reinfecção da gestante, sendo imprescindível que os tratamentos ocorram simultaneamente a fim de prevenir a transmissão vertical (1).

Há diversos motivos pelos quais os parceiros não realizam o tratamento. Uma pesquisa em cinco maternidades de Fortaleza, estado do Ceará, revelou que o não tratamento dos parceiros foi explicado em parte por recusa, justificada pelos homens por desconfiança do diagnóstico, por estarem assintomáticos ou por terem medo. Outra parte foi por ocultamento do diagnóstico pela própria gestante, por temer afastamento definitivo do parceiro, para evitar conflitos no relacionamento ou por desconhecimento da importância do tratamento (27). Nesse mesmo estudo, 4,7\% das mulheres relataram ter sofrido algum tipo de violência após a revelação do diagnóstico para o parceiro (27).

Mesmo diante da importância do tratamento do parceiro para o controle da SC, essa informação foi excluída como critério de definição de caso de SC em 2017 para fins de vigilância epidemiológica. Anteriormente, a ausência de tratamento era considerada como tratamento inadequado para sífilis materna (28).

Os resultados encontrados neste e em outros estudos mostram que as falhas no pré-natal de mulheres com sífilis estão associadas ao despreparo dos profissionais pré-natalistas, à dificuldade de adesão a protocolos assistenciais, a demoras nos resultados dos exames, à não valorização de títulos baixos de VDRL e à dificuldade na abordagem dos parceiros (29-31). O enfrentamento desse agravo precisa ser feito de forma intensiva e responsável pelos gestores e profissionais da área da saúde.

No Brasil, a taxa de abortamento como consequência da SC varia de 2,2\% no Amazonas até 5,6\% no Ceará, e o desfecho de natimorto varia de 3,3\% no Amazonas, no Distrito Federal e no Rio Grande do Sul, a 10,9\% no Ceará (4). Segundo o último boletim epidemiológico, houve menor proporção de abortamento e natimortos no Brasil em 2017 (3,5\%; 3,1\%) em comparação aos dados de Niterói (6). Assim, encontramos um percentual de natimortos pequeno (5\%), semelhante ao achado em Palmas (5,9\%), no estado do Tocantins (26). Uma questão importante a salientar é a possibilidade da ocorrência de subnotificação de óbitos por SC.

Em Niterói, a incidência de SC e a velocidade de crescimento da incidência superaram as de um estudo nacional no Brasil, de 2003 a 2008 (13); e de outras localidades brasileiras, como Belo Horizonte, de 2001 a 2008 (23); Rio Grande do Sul, de 2001 a 2012 (12); Mato Grosso, de 2001 a 2011 (14); e outras cinco unidades federadas avaliadas de 2007 a 2012 (4). As tendências mais acentuadas se relacionaram a variáveis sociodemográficas já identificadas em outros estudos brasileiros $(2,11,13$, 21-26), como cor da pele parda ou preta e baixa escolaridade. Até o momento, este é o único estudo que avaliou a tendência temporal por métodos de regressão, de acordo com características sociodemográficas e assistenciais, revelando que a SC está aumentando em grupos populacionais mais vulneráveis socialmente. A frequência de SC em Niterói, localizada em uma área urbana consolidada, com boa cobertura de atenção básica e com IDH muito alto, mostra que, mesmo em municípios com condições de vida favoráveis, existem populações mais vulneráveis do ponto de vista social, suscetíveis a agravos como a sífilis.

Pode-se, ainda, questionar a efetividade da atenção básica do município. Outros estudos mostram que a ampliação da Estratégia Saúde da Família não resultou em melhora no controle da SC $(13,32)$. É provável que os profissionais que realizam consultas de pré-natal na atenção básica não tenham conhecimento suficiente para o manejo adequado da sífilis na gestação (29-31).

As taxas elevadas e crescentes identificadas em mães adolescentes têm sido pouco relatadas no país (25). Porém, o Boletim Epidemiológico da sífilis apontou que, a cada ano, de 2007 a 2016, houve aumento da taxa de detecção de sífilis em gestantes na faixa etária de 15 a 19 anos no Brasil (8). Além disso, esse grupo populacional tem sido associado a menor cobertura do pré-natal e menor solicitação de testes para sífilis na gestação (33).

Outros países das Américas mostraram que é possível reduzir e até eliminar a transmissão vertical da sífilis $(34,35)$. O Ministério da Saúde recomenda que sejam instituídos comitês de investigação para prevenção da transmissão vertical em locais que apresentem elevado número de casos de SC (8). Além disso, são necessárias estratégias de saúde pública voltadas às populações socialmente vulneráveis, como fácil acesso a informações sobre a sífilis e sua prevenção e sobre a importância do tratamento, bem como capacitação dos profissionais de saúde para o manejo da sífilis gestacional e para o correto preenchimento da ficha de investigação.

Uma das limitações deste estudo foi o expressivo percentual de informações ignoradas, mesmo após o relacionamento dos bancos do SINAN e do SINASC. A SC é um agravo associado à vulnerabilidade social, e pode ter havido negligência no preenchimento das informações de populações mais vulneráveis. Além disso, as variáveis disponíveis nos sistemas utilizados não abrangem toda a complexidade do agravo, principalmente em relação aos aspectos qualitativos, como o motivo do não tratamento do parceiro. Por outro lado, a análise estratificada de séries temporais para cada variável sociodemográfica e do pré-natal foi inovadora e contribuiu para a melhor compreensão desse relevante problema de saúde pública no Brasil. 
Em conclusão, as iniquidades sociais se destacaram na ocorrência de SC. Observaram-se taxas muito elevadas em mulheres pretas e o aumento nas pardas, além de uma tendência temporal de aumento em mulheres jovens, principalmente adolescentes.

É necessária a capacitação dos profissionais de saúde para o manejo da sífilis gestacional, principalmente em populações socialmente vulneráveis, e a realização de trabalho educativo com usuários dos serviços de saúde, principalmente os adolescentes. Ademais, a intersetorialidade de políticas públicas sobre determinantes sociais é primordial para alcançar a meta de eliminação da SC.

Os serviços de saúde devem estar preparados para a captação precoce da gestante no pré-natal e para realizar atividades educativas sobre a sífilis. Além disso, devem ser garantidos os insumos necessários para o diagnóstico e o tratamento do agravo, bem como asseguradas a não discriminação da população e a confidencialidade dos dados dos pacientes.
Contribuição dos autores. ALSH e HK conceberam o estudo. ALSH, HK e SCF contribuíram para o desenho do estudo, a análise e a interpretação dos dados e a redação do artigo. SMSB e LAZ contribuíram na análise e na redação. ACR contribuiu na elaboração do banco de dados. Todos os autores revisaram e aprovaram a versão final.

Agradecimentos. Os autores agradecem às instituições e aos profissionais que colaboraram para a realização deste estudo. Em especial, agradecem à Coordenação de Vigilância em Saúde de Niterói (COVIG) e à Secretaria de Estado da Saúde pelo fornecimento dos bancos de dados.

\section{Conflitos de interesse. Nada declarado pelos autores.}

Declaração. As opiniões expressas no manuscrito são de responsabilidade exclusiva dos autores e não refletem necessariamente a opinião ou política da RPSP/PAJPH ou da Organização Pan-Americana da Saúde (OPAS).

\section{REFERÊNCIAS}

1. Ministério da Saúde. Protocolo clínico e diretrizes terapêuticas para atenção integral às pessoas com infecções sexualmente transmissíveis (IST). Brasília: Secretaria de Vigilância em Saúde; 2019. Disponível em: http://bvsms.saude.gov.br/bvs/publicacoes/protocolo_ clinico_diretrizes_terapeutica_atencao_integral_pessoas_infeccoes_ sexualmente_transmissiveis.pdf Acessado em 13 de dezembro de 2019.

2. Domingues RMSM, Leal MC. Incidência de sífilis congênita e fatores associados à transmissão vertical da sífilis: dados do estudo Nascer no Brasil. Cad Saude Publica. 2016;32(6):e00082415.

3. Dallé J, Baumgarten VZ, Ramos MC, Jimenez MF, Acosta L, Bumaguin DB, et al. Maternal syphilis and accomplishing sexual partner treatment: still a huge gap. Int J STD AIDS. 2017;28(9):876-80.

4. Saraceni V, Pereira GFM, Silveira MFS, Araújo MAL, Miranda AE. Vigilância epidemiológica da transmissão vertical da sífilis: dados de seis unidades federais no Brasil. Rev Panam Salud Publica. 2017;41:e44.

5. Pan American Health Organization. New generations free of HIV, syphilis, hepatitis B and Chagas disease: EMCT plus in the Americas. Washington, DC: PAHO; 2019. Disponível em: http:/ /iris.paho. org/xmlui/bitstream/handle/123456789/50993/9789275120675_ eng.pdf? sequence $=2 \&$ isAllowed $=y$ Acessado em outubro de 2019. Acessado em 13 de dezembro de 2019.

6. Pan American Health Organization. Guidance on syphilis testing in Latin America and the Caribbean: improving uptake, interpretation and quality of testing in different clinical settings. Washington, DC: PAHO; 2015. Disponível em: http://iris.paho.org/xmlui/ bitstream/handle/123456789/7706/9789275118603_eng.pdf? sequence $=1 \&$ is Allowed $=y$ Acessado em outubro de 2019 .

7. Korenromp EL, Rowley J, Alonso M, Mello MB, Wijesooriya NS, Mahiané SG, et al. Global burden of maternal and congenital syphilis and associated adverse birth outcomes-Estimates for 2016 and progress since 2012. PLoS One. 2019;14(2):e0211720.

8. Ministério da Saúde. Boletim Epidemiológico - Sífilis 2018. Brasília: Secretaria de Vigilância em Saúde, Departamento de DST, Aids e Hepatites Virais; 2018. Disponível em: http://www.aids.gov.br/ pt-br/pub/2018/boletim-epidemiologico-de-sifilis-2018 Acessado em maio de 2019.

9. Secretaria de Estado de Saúde do Rio de Janeiro (SES/RJ), Gerência de DST, HIV/AIDS, Sangue e Hemoderivados. Informe epidemiológico. Sífilis: adquirida, materna e congênita. Rio de Janeiro: Subsecretaria de Vigilância em Saúde; 2018. Disponível em: http:/ / www.riocomsaude.rj.gov.br/Publico/MostrarArquivo.aspx?C= ZDn0IcaLuWs\%3D Acessado em outubro de 2019.
10. Soeiro CM, Miranda AE, Saraceni V, Santos MC, Talhari S, Ferreira LC. Syphilis in pregnancy and congenital syphilis in Amazonas State, Brazil: an evaluation using database linkage. Cad Saude Publica. 2014;30(4):715-23.

11. Reis GJ, Barcellos C, Pedroso MM, Xavier DR. Diferenciais intraurbanos da sífilis congênita: análise preditiva por bairros do Município do Rio de Janeiro, Brasil. Cad Saude Publica. 2018;34(9):e00105517.

12. Teixeira LO, Belarmino V, Gonçalves CV, Mendoza-Sassi RA. Tendência temporal e distribuição espacial da sífilis congênita no estado do Rio Grande do Sul entre 2001 e 2012. Cienc Saude Colet. 2018;23(8):2587-97.

13. Araújo CL, Shimizu HE, Sousa AIA, Hamann EM. Incidência da sífilis congênita no Brasil e sua relação com a Estratégia Saúde da Família. Rev Saude Publica. 2012;46(3):479-86.

14. Oliveira LR, Costa MCN, Barreto FR, Pereira SM, Dourado I, Teixeira MG. Evaluation of preventive and control measures for congenital syphilis in State of Mato Grosso. Rev Soc Bras Med Trop. 2014;47(3):334-40.

15. Antunes JLF, Cardoso MRA. Uso da análise de séries temporais em estudos epidemiológicos. Epidemiol Serv Saude. 2015;24(3):565-76.

16. Programa das Nações Unidas para o Desenvolvimento. Perfil de Niterói. Brasília: Atlas do Desenvolvimento Humano no Brasil. Disponível em: http://www.atlasbrasil.org.br/2013/pt/perfil_m/ niteroi_rj Acessado em maio de 2019.

17. Ministério da Saúde. Cobertura da atenção básica. Brasília: Informação e Gestão da Atenção Básica; 2017. Disponível em: https:// egestorab.saude.gov.br/paginas/acessoPublico/relatorios/ relHistoricoCoberturaAB.xhtml Acessado em junho de 2019.

18. Camargo Jr KR, Coeli CM. Reclink: aplicativo para o relacionamento de bases de dados, implementando o método probabilistic record linkage. Cad Saude Publica. 2000;16(2):439-47.

19. Statistical Methodology and Applications Branch, Surveillance Research Program, National Cancer Institute. Joinpoint Regression Program, Version 4.4.0.0. Bethesda: National Cancer Institute; 2017.

20. DiOrio D, Kroeger K, Ross A. Social Vulnerability in Congenital Syphilis Case Mothers: Qualitative Assessment of Cases in Indiana, 2014 to 2016. Sex Transm Dis. 2018;45(7):447-51.

21. Melo NGDO, Melo Filho DA de, Ferreira LOC. Diferenciais intraurbanos de sífilis congênita no Recife, Pernambuco, Brasil (2004-2006). Epidemiol Serv Saude. 2011;20(2):213-22.

22. Fonseca SC, Oliveira LM, Almeida NMR, Silva KS, Kale PL. Incidence of congenital syphilis in a metropolitan region of Rio de Janeiro state: social inequalities. J Bras Doenças Sex Transm. 2013;25(1):22-5. 
23. Lima MG, Santos RF, Barbosa GJ, Ribeiro GS. Incidência e fatores de risco para sífilis congênita em Belo Horizonte, Minas Gerais, 20012008. Cienc Saude Colet. 2013;18(2):499-506.

24. Serafim AS, Moretti GP, Serafim GS, Niero CV, Rosa MI, Pires MMS, et al. Incidence of congenital syphilis in the South Region of Brazil. Rev Soc Bras Med Trop. 2014;47(2):170-8.

25. Nonato SM, Melo APS, Guimarães MDC. Sífilis na gestação e fatores associados à sífilis congênita em Belo Horizonte, MG, 20102013. Epidemiol Serv Saude. 2015;24(4):681-94.

26. Cavalcante PAM, Pereira RBL, Castro JGD. Syphilis in pregnancy and congenital syphilis in Palmas, Tocantins State, Brazil, 20072014. Epidemiol Serv Saude. 2017;26(2):255-64.

27. Campos AL, Araújo MAL, Melo S, Andrade RFV, Gonçalves MLC. Syphilis in parturients: aspects related to the sex partner. Rev Bras Ginecol Obstet. 2012;34(9):397-402.

28. Ministério da Saúde. Nota informativa n² - SEI/2017 -, DIAHV/ SVS/MS: altera os critérios de definição de casos para notificação de sífilis adquirida, sífilis em gestantes e sífilis congênita. Brasília: Departamento de Vigilância, Prevenção e Controle das IST, do HIV / AIDS e das DIAHV; 2017. Disponível em: http:/ / portalsinan. saude.gov.br/images / documentos/Agravos/Sifilis-Ges / Nota_Informativa_Sifilis.pdf Acessado em outubro de 2019.

29. Domingues RMSM, Hartz ZMA, Leal MC. Avaliação das ações de controle da sífilis e do HIV na assistência pré-natal da rede pública do município do Rio de Janeiro, Brasil. Rev Bras Saude Mater Infant. 2012;12(3):269-80.

30. Domingues RMSM, Lauria LM, Saraceni V, Leal MC. Manejo da sífilis na gestação: conhecimentos, práticas e atitudes dos profissionais pré-natalistas da rede SUS do município do Rio de Janeiro. Cienc Saude Colet. 2013;18(5):1341-51.

31. Rodrigues DC, Domingues RMSM. Management of syphilis in pregnancy: Knowledge and practices of health care providers and barriers to the control of disease in Teresina, Brazil. Int J Health Plann Manage. 2018;33(2):329-44.

32. Saraceni V, Miranda AE. Relação entre a cobertura da Estratégia Saúde da Família e o diagnóstico de sífilis na gestação e sífilis congênita. Cad Saude Publica. 2012;28(3):490-6.

33. Almeida AHV, Gama SGN, Costa MCO, Viellas EF, Martinelli KG, Leal MC. Desigualdades econômicas e raciais na assistência pré-natal de grávidas adolescentes, Brasil, 2011-2012. Rev Bras Saude Mater Infant. 2019;19(1):53-62.

34. Espinosa MCS, Lauzurique ME, Alcázar VRH, Pacheco BLC, Lubián MCM, Cala DC, et al. Atención a la salud maternoinfantil en Cuba: logros y desafios. Rev Panam Salud Publica. 2018;42:e27.

35. Korenromp EL, Ríos C, Apolinar ALS, Caicedo S, Cuellar D, Cárdenas I, et al. Prevalence and incidence estimates for syphilis, chlamydia, gonorrhea, and congenital syphilis in Colombia, 19952016. Rev Panam Salud Publica. 2018;42:e118.

Manuscrito recebido em 8 de julho de 2019. Aceito em versão revisada em 7 de outubro de 2019.

\section{Inequalities in congenital syphilis trends in the city of Niterói, Brazil, 2007-2016}

ABSTRACT Objective. To describe the temporal distribution and epidemiologic characteristics of congenital syphilis (CS) cases in the city of Niterói, southeastern Brazil, from 2007 to 2016.

Method. This descriptive time series analysis of the incidence of CS used data from the Notifiable Diseases Information System (SINAN) and the Live Birth Information System (SINASC). The sample included all notified cases. A probabilistic matching was performed between SINAN and SINASC data to recover ignored information. The time series was estimated using logarithmic regression according to sociodemographic and prenatal care variables.

Results. There were 754 identified cases of CS in the study period (mean incidence: 11.9 cases/1 000 live births). The incidence was higher in younger women (10 to 19; 20 to 24 years) and in those with black skin, low schooling, and without prenatal care. Of the overall group, only $57.6 \%$ received a diagnosis of syphilis during prenatal care. Treatment was not adequate in $87,7 \%$, and only $12.2 \%$ of partners were treated. SC incidence presented a growing trend of $16 \% / y e a r$, reaching 23.2 cases/1 000 living births in 2016. This growth was especially marked in female adolescents (25.2\%/year), brown race/skin color (16.8\%/year), women with low schooling (57.1\%/year) and women who received prenatal care (17.3\%/year); and, from 2012 to 2016, in women without information on skin color.

Conclusions. Social inequalities were linked to CS in the present sample. Also, increasing CS incidence was detected in youth. Health care professionals must be trained to manage gestational syphilis, and public policies must effectively address the social determinants of this condition.

Keywords Syphilis, congenital; social inequity; time series studies; surveillance; Brazil. 


\section{Desigualdades en la tendencia de sífilis congénita en la ciudad de Niterói, Brasil, 2007-2016}

RESUMEN Objetivo. Describir la distribución temporal y las características epidemiológicas de la sífilis congénita en Niterói, sureste de Brasil, de 2007 a 2016.

Métodos. Estudio descriptivo de series temporales sobre la incidencia de la sífilis congénita; se utilizaron datos del Sistema de Información de Enfermedades Notificables (SINAN) y del Sistema de Información de Nacimientos Vivos (SINASC). La muestra incluyó todos los casos reportados. Se realizó además una relación probabilística entre el SINAN y el SINASC para recuperar información ignorada. La serie temporal se estimó mediante regresión logarítmica, según variables sociodemográficas y prenatales.

Resultados. En el período estudiado se identificaron 754 casos de sífilis congénita (incidencia media de 11,9 casos/1 000 nacidos vivos). La incidencia fue mayor en las mujeres jóvenes (10-19, 20-24 años), mujeres de raza negra, con baja escolaridad y sin atención prenatal. Del total de mujeres, sólo se realizó el diagnóstico de sífilis durante la atención prenatal en $57,6 \%$. El tratamiento fue inadecuado en $87,7 \%$ de las mujeres, solo el $12,2 \%$ de las parejas fueron tratadas. La enfermedad presentó una tendencia creciente (16\%/año), y alcanzó 23,2 casos/1 000 nacidos vivos en 2016. El aumento fue más pronunciado en las adolescentes (25,2\%/año), raza/color de piel morena (16.8\%/año), mujeres con baja educación (57,1\%/año), mujeres que recibieron atención prenatal (17,3\%/año) y, de 2012 a 2016, en mujeres sin información sobre el color de la piel.

Conclusiones. En esta muestra se destacaron las desigualdades sociales en la aparición de sífilis congénita, con una incidencia creciente en las jóvenes. Se requiere capacitación de los profesionales de la salud en el manejo de la sífilis gestacional y la acción efectiva de políticas públicas sobre los determinantes sociales de la sífilis.

Palabras clave Sífilis congénita; inequidad social; estudios de series temporales; vigilancia; Brasil. 